\title{
An operational method to solve fractional differential equations
}

M. M. Rodrigues, and N. Vieira

Citation: AIP Conference Proceedings 1637, 1143 (2014); doi: 10.1063/1.4904690

View online: http://dx.doi.org/10.1063/1.4904690

View Table of Contents: http://aip.scitation.org/toc/apc/1637/1

Published by the American Institute of Physics 


\title{
An operational method to solve fractional differential equations
}

\author{
M.M. Rodrigues and N. Vieira \\ CIDMA - Center for Research and Development in Mathematics and Applications, Department of Mathematics, \\ University of Aveiro, Campus Universitário de Santiago, 3810-193 Aveiro, Portugal. \\ Email: mrodrigues@ua.pt,nloureirovieira@gmail.com
}

\begin{abstract}
In this paper, we present an operational method for solving two fractional equations, namely, the Legendre and the Laguerre equations. Based on operational approach for the Laplace and Mellin, we obtain a particular solution as a generalized power series for both equations, where the fractional derivatives are defined in the Riemann-Liouville sense. We prove the existence and uniqueness of solutions via Banach fix point theorem.
\end{abstract}

Keywords: Riemann-Liouville and Caputo derivatives; Fractional differential equations; Fractional Legendre differential equation; Fractional Laguerre differential equation; Mellin and Laplace transforms.

PACS: $02.30 . J r, 02.30 . V v$.

\section{INTRODUCTION}

The use of fractional calculus in mathematical modeling has become popular in recent years. In particular, fractional differential equations have attracted a considerable interest due to the possibility of obtain more accurate models for physical phenomena. This growing interest led to a systematics development of the analytic theory of fractional differential equations with variable coefficients, see for example the book of Samko, Kilbas and Marichev [11]. Among analytical methods, which are widely used to solve precisely fractional equations, there are methods involving integral transforms, for example, the Laplace, Fourier, and Mellin transforms (see [2, 6]). Solutions of fractional equations are usually given in the form of special functions (see $[4,7]$ ), such as the Mittag-Leffler function, the Wright function, the Fox H-function and the Meijer G-function. However, since some useful properties of the standard calculus cannot carry over analogously to the case of fractional calculus, the analytical solutions of fractional differential equations under certain initial and boundary conditions are difficult to obtain. In the recent papers $[9,10]$ we have an important application of fractional calculus techniques. Here, the authors investigated, respectively, the fractional Whittaker and Bessel equations involving Riemann-Liouville derivative, and obtained a possible solution in terms of power series by using operational calculus for the Laplace and Mellin transform. The aim of this paper is to apply an operational method to solve the fractional correspondent of two classical differential equations, namely:

- the fractional Legendre equation $\left(1-x^{2 \alpha}\right)\left(D_{0^{+}}^{2 \alpha} y\right)(x)-2 x^{\alpha}\left(D_{0^{+}}^{\alpha} y\right)(x)+\gamma(\gamma+1) y(x)=0$, where $\left.x>0, \alpha \in\right] 0,1[$ and $\gamma \in] 0,2$ [. In particular, the case $\gamma=\alpha=1$ leads us to the classical Legendre's equation (see [3]), whose solutions are, correspondingly, the Legendre polynomials.

- the fractional Laguerre equation $x^{\alpha}\left(D_{0^{+}}^{2 \alpha} l\right)(x)+\left(-x^{\alpha}+\beta+1\right)\left(D_{0^{+}}^{\alpha} l\right)(x)+\gamma l(x)=0$, where $\left.x>0, \alpha \in\right] 0,1[$, $\beta>-1$ and $\gamma \in] 0,2[$. In particular, the case $\gamma=\alpha=1$ leads us to the classical Laguerre's equation (see [3]), whose solutions are, correspondingly, the Laguerre polynomials.

We remark that, as far as the authors are aware, there were no attempt to study these general fractional equations. Only in [5] was considered a particular case of the fractional Legendre equation presented above, and in [1] fractional Laguerre polynomials $L_{\gamma}^{\beta}$ were study.

The paper is structured as follows: in the Preliminaries we recall basic properties of the Mellin transform and necessary elements of fractional calculus. In Section 3, we study the existence and uniqueness of solutions for both equations appealing to the Banach fix point theorem. In Sections 4 and 5, we present a method based on operational approach for the Laplace and Mellin for solving the fractional Legendre and Laguerre equations, respectively. 


\section{PRELIMINARIES}

In this section, we recall some basic notions about the Mellin transform and fractional calculus which we will use below. The Mellin transform is defined by (see [12]) $F(s)=M\{f(x) ; s\}=\int_{0}^{\infty} f(x) x^{s-1} d x$, where $s$ is complex, such that $\gamma_{1}<\operatorname{Re}(s)<\gamma_{2}$. This integral transform exists if $f(x)$ is piecewise continuous in every closed interval $\left.[a, b] \subset\right] 0,+\infty[$, $\int_{0}^{1}|f(x)| x^{\gamma_{1}-1} d x<\infty$ and $\int_{1}^{\infty}|f(x)| x^{\gamma_{2}-1} d x<\infty$.

If $f$ satisfies Dirichlet's condition in every closed interval $[a, b] \subset] 0,+\infty[$, then it can be restored by $f(x)=$ $\frac{1}{2 \pi i} \int_{\gamma-i \infty}^{\gamma+i \infty} M\{f(x) ; s\} x^{-s} d s, 0<x<\infty$, where $\gamma_{1}<\gamma<\gamma_{2}$.

The space of measurable functions $f$ on $] a, b\left[\right.$ such that $\int_{a}^{b}|f(x)|^{p} d x<\infty$ will be denoted by $L^{p}(a, b)$ with $p \in\left[1,+\infty\left[\right.\right.$, and for $p=1$, we write $L$ instead of $L^{1}$ (see [12]).

Theorem 1 (c.f. [12]). Let $x^{\beta-1} f(x)$ and $x^{\beta-1} g(x)$ belonging to $L(0,+\infty)$, and let $h(x)=\left(f *_{M} g\right)(x)=$ $\int_{0}^{\infty} f(y) g\left(\frac{x}{y}\right) \frac{d y}{y}$. Then $x^{\beta-1} h(x)$ belongs to $L(0,+\infty)$ and its Mellin transform is $F(s) G(s)$.

Now we recall some definitions and basic results concerning fractional calculus. Consider $n=[\beta]+1$ for $\beta \notin \mathbb{N}_{0}$ and $n=\beta$ for $\beta \in \mathbb{N}_{0}$, such that $0 \leq n-1<\beta<n$. The Riemann-Liouville fractional derivative is given by (see [4])

$$
\left(D_{0^{+}}^{\beta} f\right)(x)=\left(\frac{d}{d x}\right)^{n} \frac{1}{\Gamma(n-\beta)} \int_{0}^{x} \frac{f(t)}{(x-t)^{\beta-n+1}} d t, \quad n=[\beta]+1,
$$

where $[\beta]$ is the integer part of $\beta$. Fractional derivative (1) is defined even for some functions with a singularity at the origin, e.g., for $f(x)=x^{d}$, with $d>-1$,

$$
\left(D_{0^{+}}^{\beta} f\right)(x)=\frac{\Gamma(d+1)}{\Gamma(d+1-\beta)} x^{d-\beta}
$$

so that $D_{0^{+}}^{\beta} f=0$ if $f(x)=x^{\beta-1}$. The Caputo derivative of fractional order $\beta$ of a function $f$ is defined, in turn, as (see [4]) ${ }^{C} D_{0^{+}}^{\beta} f(x)=\frac{1}{\Gamma(n-\beta)} \int_{0}^{x}(x-\tau)^{n-\beta-1} f^{(n)}(\tau) d \tau$, where $n-1 \leq \beta<n$ and $n \in \mathbb{N}$. The Mellin transform of the Riemann-Liouville derivative has the following properties (see $[4,8,11]$ )

$$
\begin{aligned}
& M\left\{\left(D_{0^{+}}^{\beta} f\right)(x) ; s\right\}=\left.\sum_{k=0}^{n-1} \frac{\Gamma(1+k-s)}{\Gamma(1-s)}\left(D_{0^{+}}^{\beta-n} f\right)(x) x^{s-k-1}\right|_{0} ^{+\infty}+\frac{\Gamma(1-s+\beta)}{\Gamma(1-s)} F(s-\beta), \\
& M\left\{x^{\beta}\left(D_{0^{+}}^{2 \beta} f\right)(x) ; s\right\}=\frac{\Gamma(1-s+\beta)}{\Gamma(1-s-\beta)} F(s-\beta), \quad M\left\{\left(D_{0^{+}}^{\beta} f\right)(x) ; s\right\}=\frac{\Gamma(1-s+\beta)}{\Gamma(1-s)} F(s-\beta) .
\end{aligned}
$$

If $f$ is such that all our integrands terms are vanished, property (3) takes the simplified form $M\left\{\left(D_{0^{+}}^{\beta} f\right)(x) ; s\right\}=$ $\frac{\Gamma(1-s+\beta)}{\Gamma(1-s)} F(s-\beta) . A C^{n}([a, b])$ will denote the space of complex-valued functions $f$ which have continuous derivatives up to order $n-1$ on $[a, b]$ such that $f^{(n-1)} \in A C([a, b])$, where $A C([a, b])$ is the space of all functions absolutely continuous on $[a, b]$ (see [4]).

Theorem 2 (c.f. [4]) Let $\beta \geq 0$ and $v \in A C^{n}([a, b])$. Then $D_{a^{+}}^{\beta} v$ exists almost everywhere and may be represented in the form $\left(D_{a^{+}}^{\beta} v\right)(x)=\sum_{k=0}^{n-1} \frac{v^{(k)}(a)}{\Gamma(1+k-\beta)}(x-a)^{k-\beta}+\frac{1}{\Gamma(n-\beta)} \int_{a}^{x} \frac{v^{(n)}(t)}{(x-t)^{\beta-n+1}} d t$.

We remark that fractional derivative (1) verifies

$$
{ }^{C}\left(D_{a^{+}}^{\beta} v\right)(x)=\left(D_{a^{+}}^{\beta} v\right)(x)-\sum_{k=0}^{n-1} \frac{v^{(k)}(a)}{\Gamma(k-\beta+1)}(x-a)^{k-\beta} .
$$

Theorem 3 (c.f. [4]) Let $\beta \geq 0$. If $v \in A C^{n}([a, b])$ then the Caputo fractional derivative ${ }^{C} D_{a^{+}}^{\beta} v$ exists almost everywhere on $[a, b]$, and if $\beta \notin \mathbb{N}_{0}$ then ${ }^{C} D_{a^{+}}^{\beta} v$ is represented by ${ }^{C} D_{a^{+}}^{\beta} v(x)=\frac{1}{\Gamma(n-\beta)} \int_{a}^{x} \frac{v^{(n)}(t)}{(x-t)^{\beta-n+1}} d t:=\left(I_{a^{+}}^{n-\beta} D^{n} v\right)(x)$, where $D=\frac{d}{d x}$ is the ordinary derivative. 
If $\beta \notin \mathbb{N}_{0}$ and $n=[\beta]+1$, then (see [4])

$$
\left|\left(I_{a^{+}}^{n-\beta} D^{n} v\right)(x)\right| \leq \frac{\left\|v^{(n)}\right\|_{C}}{\Gamma(n-\beta)(n-\beta+1)}(x-a)^{n-\beta},
$$

where $\left\|v^{(n)}\right\|_{C}=\max _{x \in[a, b]}\left|v^{(n)}(x)\right|$, and the Riemann-Liouville fractional integral $I_{a^{+}}^{n-\beta}$ is defined by (see [4]) $\left(I_{a^{+}}^{\alpha} f\right)(x)=\frac{1}{\Gamma(\alpha)} \int_{a}^{x} \frac{f(t)}{(x-t)^{1-\alpha}} d t, x>a, \operatorname{Re}(\alpha)>0$.

Lemma 4 (c.f. [4]) Let $\beta>0$. If $v \in A C^{n}([a, b])$ or $v \in C^{n}([a, b])$ then $\left(I_{a^{+}}^{\alpha}{ }^{C} D_{a^{+}}^{\beta} v\right)(x)=v(x)-\sum_{k=0}^{n-1} \frac{v^{(k)}(a)}{k !}(x-a)^{k}$.

Lemma 5 (c.f. [11]) If the series $f(x)=\sum_{n=0}^{\infty} f_{n}(x)$, with $f_{n} \in C([a, b])$, is uniformly convergent on $[a, b]$, then its termwise fractional integration is admissible $\left(I_{a^{+}}^{\beta} \sum_{n=0}^{\infty} f_{n}\right)(x)=\sum_{n=0}^{\infty}\left(I_{a^{+}}^{\beta} f_{n}\right)(x), \beta>0, a<x<b$, the series on the right-hand side being also uniformly convergent on $[a, b]$.

Lemma 6 (c.f. [11]) If the fractional derivatives $D_{a^{+}}^{\beta} f_{n}$ exist for all $n \in \mathbb{N}_{0}$ and the series $\sum_{n=0}^{\infty} f_{n}, \sum_{n=0}^{\infty} D_{a^{+}}^{\beta} f_{n}$ are uniformly convergent on every sub-interval $[a+\varepsilon, b], \varepsilon>0$. Then the former series admits termwise fractional differentiation using the formula $\left(D_{a^{+}}^{\beta} \sum_{n=0}^{\infty} f_{n}\right)(x)=\left(\sum_{n=0}^{\infty} D_{a^{+}}^{\beta} f_{n}\right)(x), \beta>0, a<x<b$.

\section{EXISTENCE AND UNIQUENESS OF SOLUTIONS}

\section{Fractional Legendre equation}

Here we will use the Banach's fixed point theorem to study the existence and uniqueness of solutions for the fractional Legendre equation

$$
\left(1-x^{2 \alpha}\right)\left(D_{0^{+}}^{2 \alpha} y\right)(x)-2 x^{\alpha}\left(D_{0^{+}}^{\alpha} y\right)(x)+\gamma(\gamma+1) y(x)=0,
$$

with $\left.x \in\left[0, X_{0}\right], X_{0}>1, \alpha \in\right] 0,1[$ and $\gamma \in] 0,2[$, under the initial conditions

$$
y(0)=y_{0}, \quad y^{\prime}(0)=y_{0}^{*} .
$$

Let $I=[a, b],(a<b, a, b \in \mathbb{R})$ and $m \in \mathbb{N}_{0}$. Denote by $C^{m}$ the usual space of functions $v$ which are $m$ times continuously differentiable on $I$, with the norm $\|v\|_{C^{m}}=\sum_{k=0}^{m}|| v^{(k)} \|_{C}=\sum_{k=0}^{m} \max _{x \in I}\left|v^{(k)}(x)\right|$. In particular for $m=0, C^{0}(I) \equiv C(I)$ is the space of continuous functions $v$ on $I$ with the norm $\|v\|_{C}=\max _{x \in I}|v(x)|$.

Theorem 7 The fractional problem (7-8), has a unique solution for every $\alpha \in] 0,1[$ if

$$
\gamma(\gamma+1)>\frac{X_{0}^{2}+1}{(3-2 \alpha) \Gamma(2(1-\alpha))}+\frac{2 X_{0}}{(2-\alpha) \Gamma(1-\alpha)}
$$

and $\gamma \in] 0,2[$.

Proof: Consider the Banach spaces $X=\left\{y: y(x) \in C^{2}\left(\left[0, X_{0}\right]\right)\right\}$ and $Y=\left\{y: y(x) \in C\left(\left[0, X_{0}\right]\right)\right\}$. Putting $T: X \rightarrow Y$, $(T y)(x)=\frac{1}{\gamma(\gamma+1)}\left[\left(x^{2 \alpha}-1\right)\left(D_{0^{+}}^{2 \alpha} y\right)(x)+2 x^{\alpha}\left(D_{0^{+}}^{\alpha} y\right)(x)\right]$, we rewrite equation (7) in the form $y(x)=(T y)(x)$. Taking into account Theorems 2, 3, Lemma 4 and relations (5), (6), we have

$$
\begin{aligned}
\left\|T y_{1}-T y_{2}\right\|_{Y} & =\frac{1}{\gamma(\gamma+1)}\left\|\left(x^{2 \alpha}-1\right)\left(D_{0^{+}}^{2 \alpha}\left(y_{1}-y_{2}\right)\right)(x)+2 x^{\alpha}\left(D_{0^{+}}^{\alpha}\left(y_{1}-y_{2}\right)\right)(x)\right\|_{Y} \\
& \leq \frac{1}{\gamma(\gamma+1)}\left(\frac{X_{0}^{2}+1}{(3-2 \alpha) \Gamma(2(1-\alpha))}\left\|D^{2}\left(y_{1}-y_{2}\right)\right\|_{Y}+\frac{2 X_{0}}{(2-\alpha) \Gamma(1-\alpha)}\left\|D\left(y_{1}-y_{2}\right)\right\|_{Y}\right) \\
& \leq \frac{1}{\gamma(\gamma+1)}\left(\frac{X_{0}^{2}+1}{(3-2 \alpha) \Gamma(2(1-\alpha))}+\frac{2 X_{0}}{(2-\alpha) \Gamma(1-\alpha)}\right)\left\|y_{1}-y_{2}\right\|_{X}
\end{aligned}
$$

From (9) we conclude that $T$ is a contraction. Hence we can apply the Banach fix point theorem to complete the proof. 


\section{Fractional Laguerre equation}

Here we will use the Banach's fixed point theorem to study the existence and uniqueness of solutions for the fractional Laguerre equation

$$
x^{\alpha}\left(D_{0^{+}}^{2 \alpha} w\right)(x)+\left(-x^{\alpha}+\beta+1\right)\left(D_{0^{+}}^{\alpha} w\right)(x)+\gamma w(x)=0
$$

with $\left.x \in\left[0, X_{0}\right], X_{0}>1, \alpha \in\right] 0,1[, \beta>-1$ and $\gamma \in] 0,2[$, under the initial conditions

$$
l(0)=l_{0}, \quad l^{\prime}(0)=l_{0}^{*} .
$$

Let $I=[a, b],(a<b, a, b \in \mathbb{R})$ and $m \in \mathbb{N}_{0}$. Denote by $C^{m}$ the usual space of functions $v$ which are $m$ times continuously differentiable on $I$, with the norm $\|v\|_{C^{m}}=\sum_{k=0}^{m}\left\|v^{(k)}\right\|_{C}=\sum_{k=0}^{m} \max _{x \in I}\left|v^{(k)}(x)\right|$. In particular for $m=0, C^{0}(I) \equiv C(I)$ is the space of continuous functions $v$ on $I$ with the norm $\|v\|_{C}=\max _{x \in I}|v(x)|$.

Theorem 8 The fractional problem (10-11), has a unique solution for every $\alpha \in] 0,1[, \beta>-1$ if

$$
\gamma>\left[\frac{X_{0}}{(3-2 \alpha) \Gamma(2(1-\alpha))}+\frac{X_{0}+\beta+1}{(2-\alpha) \Gamma(1-\alpha)}\right]
$$

and $\gamma \in] 0,2[$.

Proof: Consider the Banach spaces $X=\left\{l: l(x) \in C^{2}\left(\left[0, X_{0}\right]\right)\right\}$ and $Y=\left\{l: l(x) \in C\left(\left[0, X_{0}\right]\right)\right\}$. Putting $T: X \rightarrow Y$, $(T l)(x)=\frac{1}{\gamma}\left[-x^{\alpha}\left(D_{0^{+}}^{2 \alpha} l\right)(x)+\left(x^{\alpha}-\beta-1\right)\left(D_{0^{+}}^{\alpha} l\right)(x)\right]$, we rewrite equation (10) in the form $l(x)=(T l)(x)$. Taking into account Theorems 2, 3, Lemma 4 and relations (5), (6), we have

$$
\begin{aligned}
\left\|T l_{1}-T l_{2}\right\|_{Y} & =\frac{1}{\gamma}\left\|-x^{\alpha}\left(D_{0^{+}}^{2 \alpha}\left(l_{1}-l_{2}\right)\right)(x)+\left(x^{\alpha}-\beta-1\right)\left(D_{0^{+}}^{\alpha}\left(l_{1}-l_{2}\right)\right)(x)\right\|_{Y} \\
& \leq \frac{1}{\gamma}\left[\frac{X_{0}}{(3-2 \alpha) \Gamma(2(1-\alpha))}\left\|D^{2}\left(l_{1}-l_{2}\right)\right\|_{Y}+\frac{X_{0}+\beta+1}{(2-\alpha) \Gamma(1-\alpha)}\left\|D\left(l_{1}-l_{2}\right)\right\|_{Y}\right] \\
& \leq \frac{1}{\gamma}\left[\frac{X_{0}}{(3-2 \alpha) \Gamma(2(1-\alpha))}+\frac{X_{0}+\beta+1}{(2-\alpha) \Gamma(1-\alpha)}\right]\left\|l_{1}-l_{2}\right\|_{X}
\end{aligned}
$$

From (12) we conclude that $T$ is a contraction. Hence we can apply the Banach fix point theorem to complete the proof.

\section{AN OPERATIONAL METHOD TO SOLVE FRACTIONAL LEGENDRE EQUATION}

The aim of this section is to obtain a particular solutions for the fractional Legendre equation (7), i.e., (1 $\left.x^{2 \alpha}\right)\left(D_{0^{+}}^{2 \alpha} y\right)(x)-2 x^{\alpha}\left(D_{0^{+}}^{\alpha} y\right)(x)+\gamma(\gamma+1) y(x)=0$, where $\left.x>0, \alpha \in\right] 0,1[, \gamma \in] 0,2\left[, x \in\left[x_{0}, X_{0}\right], x_{0}, X_{0} \in \mathbb{R}^{+}\right.$, and $D_{0^{+}}^{2 \alpha}, D_{0^{+}}^{\alpha}$ are the operators of the Riemann fractional derivative (1).

We will apply a method to obtain an approximate solution of the fractional Legendre equation by using the direct and inverse Mellin transforms. Hence applying Mellin's transform to (7) and taking into account properties (4), we have

$$
-\frac{\Gamma(1-s)}{\Gamma(1-s-2 \alpha)} Y(s)+Y(s-2 \alpha)-\frac{2 \Gamma(1-s)}{\Gamma(1-s-\alpha)} Y(s)+\gamma(\gamma+1) Y(s)=0 .
$$

Denoting $H(s)=\Gamma(1-s) Y(s)$, where $Y(s)$ is the Mellin transform of $y(x)$ and

$$
h(x)=\int_{0}^{\infty} e^{-x t} y(t) d t
$$

we get owing to Theorem 1 that $h(x)$ is the inverse Mellin transform of $H(s)$. Thus (13) became

$$
-H(s)+H(s-2 \alpha)-\frac{2 \Gamma(1-s-2 \alpha)}{\Gamma(1-s-\alpha)} H(s)+\gamma(\gamma+1) \frac{\Gamma(1-s-2 \alpha)}{\Gamma(1-s)} H(s)=0 .
$$


Taking the inverse Mellin transform we obtain, correspondingly, the equality

$$
-h(x)+x^{-2 \alpha} h(x)-2 \int_{0}^{\infty} h\left(\frac{x}{t}\right) k_{1}(t) \frac{d t}{t}+\gamma(\gamma+1) \int_{0}^{\infty} h\left(\frac{x}{t}\right) k_{2}(t) \frac{d t}{t}=0
$$

where

$$
\begin{aligned}
& k_{1}(t)=M^{-1}\left\{\frac{\Gamma(1-s-2 \alpha)}{\Gamma(1-s-\alpha)}\right\}=\frac{t^{\alpha}}{\Gamma(\alpha)}(t-1)_{+}^{\alpha-1}=\left\{\begin{array}{ll}
\frac{t^{\alpha}(t-1)^{\alpha-1}}{\Gamma(\alpha)}, & t>1 \\
0, & t<1
\end{array} ;\right. \\
& k_{2}(t)=M^{-1}\left\{\frac{\Gamma(1-s-2 \alpha)}{\Gamma(1-s)}\right\}=\frac{1}{\Gamma(2 \alpha)}(t-1)_{+}^{2 \alpha-1}=\left\{\begin{array}{ll}
\frac{(t-1)^{2 \alpha-1}}{\Gamma(2 \alpha)}, & t>1 \\
0, & t<1
\end{array} .\right.
\end{aligned}
$$

Consider that $h(x)$ admits formal series representation $h(x) \sim \sum_{n=1}^{\infty} b_{n} x^{-\alpha n}$, i.e.,

$$
h(x)=\sum_{n=1}^{N} b_{n} x^{-\alpha n}+O\left(x^{-\alpha N}\right), \quad x \rightarrow \infty, N \in \mathbb{N} .
$$

Substituting (19) into (16) and using Lemma 6 with (17) and (18), we come out with the asymptotic equality

$$
\begin{aligned}
-\sum_{n=1}^{N} b_{n} x^{-\alpha n}+x^{-2 \alpha} \sum_{n=1}^{N} b_{n} x^{-\alpha n}-\frac{2}{\Gamma(\alpha)} \sum_{n=1}^{N} b_{n} x^{-\alpha n} \int_{1}^{\infty} t^{\alpha n-1}(t-1)^{\alpha-1} d t & \\
& +\frac{\gamma(\gamma+1)}{\Gamma(2 \alpha)} \sum_{n=1}^{N} b_{n} x^{-\alpha n} \int_{1}^{\infty} t^{\alpha n-1}(t-1)^{2 \alpha-1} d t=O\left(x^{-\alpha N}\right) .
\end{aligned}
$$

At the meantime, the elementary Beta-integrals that appear in (20) are calculated explicitly under condition $\alpha<\frac{1}{N+2}$.

$$
\begin{array}{ll}
\int_{1}^{\infty} t^{\alpha n-1}(t-1)^{\alpha-1} d t=\frac{\Gamma(\alpha) \Gamma(-\alpha(n+1)+1)}{\Gamma(-\alpha n+1)}, & n=0, \ldots, N . \\
\int_{1}^{\infty} t^{\alpha n-1}(t-1)^{2 \alpha-1} d t=\frac{\Gamma(2 \alpha) \Gamma(-\alpha(n+2)+1)}{\Gamma(-\alpha n+1)}, & n=0, \ldots, N .
\end{array}
$$

Therefore, substituting (21) and (22) into (20), we arrive at the following truncated equation

$$
\begin{aligned}
& -\sum_{n=1}^{N} b_{n} x^{-\alpha n}+x^{-2 \alpha} \sum_{n=1}^{N} b_{n} x^{-\alpha n} \\
& \quad+2 \sum_{n=1}^{N} b_{n} \frac{\Gamma(-\alpha(n+1)+1)}{\Gamma(-\alpha n+1)} x^{-\alpha n}+\gamma(\gamma+1) \sum_{n=1}^{N} b_{n} \frac{\Gamma(-\alpha(n+2)+1)}{\Gamma(-\alpha n+1)} x^{-\alpha n}=0 .
\end{aligned}
$$

Collecting the terms which contain equal power of $x$ and equating them to zero, we find

$$
\left\{\begin{array}{l}
b_{1}\left[2 \frac{\Gamma(-2 \alpha+1)}{\Gamma(-\alpha+1)}+\gamma(\gamma+1) \frac{\Gamma(-3 \alpha+1)}{\Gamma(-\alpha+1)}-1\right]=0 \\
b_{2}\left[2 \frac{\Gamma(-3 \alpha+1)}{\Gamma(-2 \alpha+1)}+\gamma(\gamma+1) \frac{\Gamma(-4 \alpha+1)}{\Gamma(-2 \alpha+1)}-1\right]=0 \\
b_{n}\left[2 \frac{\Gamma(-\alpha(n+1)+1)}{\Gamma(-\alpha n+1)}+\gamma(\gamma+1) \frac{\Gamma(-\alpha(n+2)+1)}{\Gamma(-\alpha n+1)}-1\right]+b_{n-2}=0, \quad n=3,4,5, \ldots, N
\end{array}\right.
$$

Numerical simulations indicate that $\gamma^{2}+\gamma \neq \frac{\Gamma(-\alpha n+1)-2 \Gamma(-\alpha(n+1)+1)}{\Gamma(-\alpha(n+2)+1)}$, with $\left.\left.\left.\alpha \in\right] 0, \frac{1}{N+2}\right], \gamma \in\right] 0,2[, n=3,4,5, \ldots, N$. This means, from the previous system, that the case $b_{1}=b_{2}=0$ drives to the trivial solution of equation (7). From the second term of the left hand side of the first and second equations of (23), we have the following equation

$$
\frac{\Gamma(-4 \alpha+1)[2 \Gamma(-2 \alpha+1)-\Gamma(-\alpha+1)]}{\Gamma(-3 \alpha+1)[2 \Gamma(-3 \alpha+1)-\Gamma(-2 \alpha+1)]}=1 .
$$

Considering numerical simulations, we have the indications that (24) has a unique solution, namely $\alpha_{1}$, in the interval ]0,80;0,85[. This fact implies the necessity of split the study of (23) in two main cases 


\section{Case I}

Consider that $\alpha \neq \alpha_{1}$. This implies that $b_{1}$ and $b_{2}$ cannot be nonzero simultaneously as well. So let for instance $b_{1} \neq 0$. Consequently $\gamma(\gamma+1)=\frac{\Gamma(-\alpha+1)-2 \Gamma(-2 \alpha+1)}{\Gamma(-3 \alpha+1)}$. Hence the third equation of (23) becomes $b_{n}\left[2 \frac{\Gamma(-\alpha(n+1)+1)}{\Gamma(-\alpha n+1)}+\frac{\Gamma(-\alpha(n+2)+1)[\Gamma(-\alpha+1)-2 \Gamma(-2 \alpha+1)]}{\Gamma(-\alpha n+1) \Gamma(-3 \alpha+1)}-1\right]+b_{n-2}=0$, with $n=3,4,5, \ldots, N$. Furthermore, numerical simulations confirm $2 \frac{\Gamma(-\alpha(n+1)+1)}{\Gamma(-\alpha n+1)}+\frac{\Gamma(-\alpha(n+2)+1)[\Gamma(-\alpha+1)-2 \Gamma(-2 \alpha+1)]}{\Gamma(-\alpha n+1) \Gamma(-3 \alpha+1)}-1 \neq 0$, for $\left.\left.\alpha \neq \alpha_{1}, \alpha \in\right] 0, \frac{1}{N+2}\right]$, and $n=3,4,5 \ldots, N$. Therefore one can express coefficients $b_{n}$ with odd indices by the relation

$$
b_{2 n+1}=(-1)^{n} b_{1} \prod_{k=1}^{n}\left[\frac{2 \Gamma(-2 \alpha(k+1)+1)}{\Gamma(-\alpha(2 k+1)+1)}+\frac{\Gamma(-\alpha(2 k+3)+1)[\Gamma(-\alpha+1)-2 \Gamma(-2 \alpha+1)]}{\Gamma(-\alpha(2 k+1)+1) \Gamma(-3 \alpha+1)}-1\right]^{-1} .
$$

Since in this case $b_{2}=0$, we have, from the third equation of (23), that all even coefficients are zero. We get an approximate solution of equation (20) in the form $h(x) \sim \sum_{n=1}^{\infty} b_{2 n+1} x^{-\alpha(2 n+1)}$. We obtain the corresponding expression for the solution $y(t)$ employing operational relation for the Laplace transform. Namely equality (14) gives $y(t)$ as a formal series

$$
y(t) \sim \sum_{n=0}^{\infty} \frac{b_{2 n+1}}{\Gamma(\alpha n)} t^{\alpha n-1}
$$

We summarize the above discussion as

Theorem 9 Let $\left.\left.b_{1} \neq 0, \alpha \in\right] 0, \frac{1}{N+2}\right], \alpha \neq \alpha_{1}, N \in \mathbb{N}_{0}, x \in\left[x, X_{0}\right], x_{0}, X_{0} \in \mathbb{R}_{+}$, and $\left.\gamma \in\right] 0,2[$ such that $\gamma(\gamma+1)=$ $\frac{\Gamma(-\alpha+1)-2 \Gamma(-2 \alpha+1)}{\Gamma(-3 \alpha+1)}$. Then the fractional Legendre equation (7) admits as a particular solution the power series (26), with the coefficients given by formula (25).

In the even case, we presume $b_{2} \neq 0$ and the determining equation for $\gamma$ becomes $\gamma(\gamma+1)=\frac{\Gamma(-2 \alpha+1)-2 \Gamma(-3 \alpha+1)}{\Gamma(-4 \alpha+1)}$. Then, from the third equation of (23) $b_{n}\left[2 \frac{\Gamma(-\alpha(n+1)+1)}{\Gamma(-\alpha n+1)}+\frac{\Gamma(-\alpha(n+2)+1)[\Gamma(-2 \alpha+1)-2 \Gamma(-3 \alpha+1)]}{\Gamma(-\alpha n+1) \Gamma(-4 \alpha+1)}-1\right]+b_{n-2}=0$. As above, numerical simulations indicate that $2 \frac{\Gamma(-\alpha(n+1)+1)}{\Gamma(-\alpha n+1)}+\frac{\Gamma(-\alpha(n+2)+1)[\Gamma(-2 \alpha+1)-2 \Gamma(-3 \alpha+1)]}{\Gamma(-\alpha n+1) \Gamma(-4 \alpha+1)}-1 \neq 0$, for $\alpha \neq \alpha_{1}$, $\left.\alpha \in] 0, \frac{1}{N+2}\right]$, and $n=3,4,5 \ldots, N$. Hence in the same manner, we express the even coefficients by the relation

$$
b_{2 n}=(-1)^{n} b_{2} \prod_{k=1}^{n}\left[2 \frac{\Gamma(-\alpha(2 k+3)+1)}{\Gamma(-2 \alpha(k+1)+1)}+\frac{\Gamma(-2 \alpha(k+2)+1)[\Gamma(-2 \alpha+1)-2 \Gamma(-3 \alpha+1)]}{\Gamma(-2 k \alpha+1) \Gamma(-4 \alpha+1)}-1\right]^{-1} .
$$

Since in this case $b_{1}=0$, we have, from the third equation of (23), that all odd coefficients are zero. We get an approximate solution of equation (20) in the form $h(x) \sim \sum_{n=1}^{\infty} b_{2 n} x^{2 n \alpha}$. Similarly to the case of the even coefficients, we obtain the corresponding expression for the solution $y(t)$ employing operational relation for the Laplace transform. Namely equality (14) gives $y(t)$ as a formal series

$$
y(t) \sim \sum_{n=1}^{\infty} \frac{b_{2 n}}{\Gamma(\alpha n)} t^{\alpha n-1}
$$

We summarize the above discussion as

Theorem 10 Let $\left.\left.b_{2} \neq 0, \alpha \in\right] 0, \frac{1}{N+2}\right], \alpha \neq \alpha_{1}, N \in \mathbb{N}_{0}, x \in\left[x, X_{0}\right], x_{0}, X_{0} \in \mathbb{R}_{+}$, and $\left.\gamma \in\right] 0,2[$ such that $\gamma(\gamma+1)=$ $\frac{\Gamma(-2 \alpha+1)-2 \Gamma(-3 \alpha+1)}{\Gamma(-4 \alpha+1)}$. Then the fractional Legendre equation (7) admits as a particular solution the power series (28), with the coefficients given by formula (27). 


\section{Case II}

Consider now that $\left.\alpha=\alpha_{1} \in\right] 0,80 ; 0,85$ [, i.e., from (24)

$$
\begin{aligned}
& \frac{\Gamma\left(-\alpha_{1}+1\right)-2 \Gamma\left(-2 \alpha_{1}+1\right)}{\Gamma\left(-3 \alpha_{1}+1\right)}=\gamma(\gamma+1)=\frac{\Gamma\left(-2 \alpha_{1}+1\right)-2 \Gamma\left(-3 \alpha_{1}+1\right)}{\Gamma\left(-4 \alpha_{1}+1\right)} \\
& \Leftrightarrow \gamma(\gamma+1)=\frac{\Gamma\left(-4 \alpha_{1}+1\right)\left[2 \Gamma\left(-2 \alpha_{1}+1\right)-\Gamma\left(-\alpha_{1}+1\right)\right]}{\Gamma\left(-3 \alpha_{1}+1\right)\left[2 \Gamma\left(-3 \alpha_{1}+1\right)-\Gamma\left(-2 \alpha_{1}+1\right)\right]} .
\end{aligned}
$$

Numerical simulations indicate that there exists $\gamma \in] 0,2[$ such that (29) is fulfilled. Comparing with Case I, we have the additional possibility of $b_{1}$ and $b_{2}$ being nonzero simultaneously. Supposing that, the third equation of (23) become $b_{n}\left[2 \frac{\Gamma\left(-\alpha_{1}(n+1)+1\right)}{\Gamma\left(-\alpha_{1} n+1\right)}+\frac{\Gamma\left(-\alpha_{1}(n+2)+1\right) \Gamma\left(-4 \alpha_{1}+1\right)\left[2 \Gamma\left(-2 \alpha_{1}-1\right)-\Gamma\left(-\alpha_{1}+1\right)\right]}{\Gamma\left(-\alpha_{1} n+1\right) \Gamma\left(-3 \alpha_{1}+1\right)\left[2 \Gamma\left(-3 \alpha_{1}+1\right)-\Gamma\left(-2 \alpha_{1}+1\right)\right]}-1\right]+b_{n-2}=0$, with $n=3,4,5, \ldots, N$. Furthermore, numerical simulations show that $2 \frac{\Gamma\left(-\alpha_{1}(n+1)+1\right)}{\Gamma\left(-\alpha_{1} n+1\right)}+\frac{\Gamma\left(-\alpha_{1}(n+2)+1\right) \Gamma\left(-4 \alpha_{1}+1\right)\left[2 \Gamma\left(-2 \alpha_{1}-1\right)-\Gamma\left(-\alpha_{1}+1\right)\right]}{\Gamma\left(-\alpha_{1} n+1\right) \Gamma\left(-3 \alpha_{1}+1\right)\left[2 \Gamma\left(-3 \alpha_{1}+1\right)-\Gamma\left(-2 \alpha_{1}+1\right)\right]}-1 \neq 0$, for $n=3,4,5, \ldots, N$. Therefore one can express coefficients $b_{n}$ with odd and even coefficients, respectively, by the relations

$$
\begin{aligned}
& b_{2 n+1}=(-1)^{n} b_{1} \\
& \quad \times \prod_{k=1}^{n}\left[\frac{2 \Gamma\left(-2 \alpha_{1}(k+1)+1\right)}{\Gamma\left(-\alpha_{1}(2 k+1)+1\right)}+\frac{\Gamma\left(-\alpha_{1}(2 k+3)+1\right) \Gamma\left(-4 \alpha_{1}+1\right)\left[2 \Gamma\left(-2 \alpha_{1}-1\right)-\Gamma\left(-\alpha_{1}+1\right)\right]}{\Gamma\left(-\alpha_{1}(2 k+1)+1\right) \Gamma\left(-3 \alpha_{1}+1\right)\left[2 \Gamma\left(-3 \alpha_{1}+1\right)-\Gamma\left(-2 \alpha_{1}+1\right)\right]}-1\right]^{-1} \\
& b_{2 n}=(-1)^{n} b_{2} \\
& \quad \times \prod_{k=1}^{n}\left[2 \frac{\Gamma\left(-\alpha_{1}(2 k+3)+1\right)}{\Gamma\left(-2 \alpha_{1}(k+1)+1\right)}+\frac{\Gamma\left(-2 \alpha_{1}(k+2)+1\right) \Gamma\left(-4 \alpha_{1}+1\right)\left[2 \Gamma\left(-2 \alpha_{1}-1\right)-\Gamma\left(-\alpha_{1}+1\right)\right]}{\Gamma\left(-2 k \alpha_{1}+1\right) \Gamma\left(-3 \alpha_{1}+1\right)\left[2 \Gamma\left(-3 \alpha_{1}+1\right)-\Gamma\left(-2 \alpha_{1}+1\right)\right]}-1\right]^{-1}
\end{aligned}
$$

We get an approximate solution of equation (20) in the form $h(x) \sim \sum_{n=1}^{\infty}\left[b_{2 n+1} x^{-\alpha_{1}(2 n+1)}+b_{2 n} x^{2 n \alpha_{1}}\right]$. Similarly to the previous cases, we obtain the corresponding expression for the solution $y(t)$ employing operational relation for the Laplace transform. Namely equality (14) gives $y(t)$ as a formal series

$$
y(t) \sim \sum_{n=1}^{\infty} \frac{b_{2 n+1}+b_{2 n}}{\Gamma\left(\alpha_{1} n\right)} t^{\alpha_{1} n-1} .
$$

We summarize the above discussion as

Theorem 11 Let $\alpha=\alpha_{1}, N \in \mathbb{N}_{0}, x \in\left[x, X_{0}\right], x_{0}, X_{0} \in \mathbb{R}_{+}$, and $\left.\gamma \in\right] 0,2\left[\right.$ such that $\gamma(\gamma+1)=\frac{\Gamma\left(-4 \alpha_{1}+1\right)\left[2 \Gamma\left(-2 \alpha_{1}-1\right)-\Gamma\left(-\alpha_{1}+1\right)\right]}{\Gamma\left(-3 \alpha_{1}+1\right)\left[2 \Gamma\left(-3 \alpha_{1}+1\right)-\Gamma\left(-2 \alpha_{1}+1\right)\right]}$. Then the fractional Legendre equation (7) admits as a particular solution the power series (32), with the coefficients given by formulas (30) and (31).

\section{AN OPERATIONAL METHOD TO SOLVE FRACTIONAL LAGUERRE EQUATION}

The aim of this section is to obtain particular solutions for the fractional Laguerre equation (10), i.e., $x^{\alpha}\left(D_{0^{+}}^{2 \alpha} l\right)(x)+$ $\left(-x^{\alpha}+\beta+1\right)\left(D_{0^{+}}^{\alpha} l\right)(x)+\gamma l(x)=0$, where $\left.x>0, \alpha \in\right] 0,1[, \beta>-1, \gamma \in] 0,2\left[, x \in\left[x_{0}, X_{0}\right], x_{0}, X_{0} \in \mathbb{R}^{+}\right.$, and $D_{0^{+}}^{2 \alpha}, D_{0^{+}}^{\alpha}$ are the operators of the Riemann fractional derivative (1).

\section{Recurrence relation for the coefficients of the series solution}

Here, we derive a recurrence relation for coefficients of the series solution associated to (10), and after that we construct solutions for the Laguerre fractional equation in terms of power series in increasing powers of argument $x$

$$
l(x)=\sum_{n=0}^{\infty} a_{n} x^{\alpha n}
$$


such that the following condition holds

$$
\sum_{n=0}^{\infty}\left|a_{n}\right| n^{2} X_{0}^{n}<\infty
$$

By Stirling's asymptotic formula for Gamma function (see [11]), we have $\frac{\Gamma(\alpha n+1)}{\Gamma(\alpha(n-2)+1)}=O\left(n^{2 \alpha}\right)$, when $n \rightarrow \infty$. Therefore, from relation (2), condition (34) and Lemma 4 we can guarantee the absolute and uniform convergence on $\left[x_{0}, X_{0}\right]$ of the corresponding series in Lemma 6. Hence we get

$$
\left(D_{0^{+}}^{\alpha} l\right)(x)=\sum_{n=0}^{\infty} a_{n} \frac{\Gamma(\alpha n+1)}{\Gamma(\alpha(n-1)+1)} x^{\alpha(n-1)}, \quad\left(D_{0^{+}}^{2 \alpha} l\right)(x)=\sum_{n=0}^{\infty} a_{n} \frac{\Gamma(\alpha n+1)}{\Gamma(\alpha(n-2)+1)} x^{\alpha(n-2)} .
$$

Substituting expressions (33) and (35) in (10), and collecting the terms containing equal powers of $x$, we derive

$$
\begin{aligned}
& \sum_{n=0}^{\infty} a_{n} \frac{\Gamma(\alpha n+1)}{\Gamma(\alpha(n-2)+1)} x^{\alpha(n-1)}-\sum_{n=1}^{\infty} a_{n-1} \frac{\Gamma(\alpha(n-1)+1)}{\Gamma(\alpha(n-2)+1)} x^{\alpha(n-1)} \\
& +(\beta+1) \sum_{n=0}^{\infty} a_{n} \frac{\Gamma(\alpha n+1)}{\Gamma(\alpha(n-1)+1)} x^{\alpha(n-1)}+\gamma \sum_{n=1}^{\infty} a_{n-1} x^{\alpha(n-1)}=0 .
\end{aligned}
$$

Evidently all the coefficients of $x^{\alpha n}$ should be equal to zero. Hence we obtain the following recurrence relations

$$
\left\{\begin{array}{l}
a_{0}\left(\frac{1}{\Gamma(1-2 \alpha)}+\frac{\beta+1}{\Gamma(1-\alpha)}\right)=0 \\
a_{n}\left(\frac{\Gamma(\alpha n+1)}{\Gamma(\alpha(n-2)+1)}+\frac{(\beta+1) \Gamma(\alpha n+1)}{\Gamma(\alpha(n-1)+1)}\right)+a_{n-1}\left(\gamma-\frac{\Gamma(\alpha(n-1)+1)}{\Gamma(\alpha(n-2)+1)}\right)=0, \quad n \in \mathbb{N}
\end{array} .\right.
$$

It is immediate from the first equation of system (37) that the case $a_{0}=0$ leads to the trivial solution of equation (10). In order to obtain non-trivial solutions, we assume that $a_{0} \neq 0$ and from the first equation of the system we obtain the following equation

$$
\frac{1}{\Gamma(1-2 \alpha)}+\frac{\beta+1}{\Gamma(1-\alpha)}=0
$$

Numerical simulations indicate that equation (38) is possible for some $\alpha \in] 0,1[$ and $\beta>-1$. Hence, considering $\alpha$ and $\beta$ such that (38) is fulfilled and taking into account the properties of Gamma function (see [3]), we get from the second equation of (37) $a_{n}=-\frac{\Gamma(\alpha(n-1)+1)[\gamma-\Gamma(\alpha(n-1)+1)]}{\Gamma(\alpha n+1)[\Gamma(\alpha(n-1)+1)+(1+\beta) \Gamma(\alpha(n-2)+1)]} a_{n-1}, n \in \mathbb{N}$. Therefore one can express the coefficients $a_{n}$ by the relation

$$
a_{n}=(-1)^{n} \prod_{k=1}^{n} \frac{\Gamma(\alpha(k-1)+1)[\gamma-\Gamma(\alpha(k-1)+1)]}{\Gamma(\alpha k+1)[\Gamma(\alpha(k-1)+1)+(1+\beta) \Gamma(\alpha(k-2)+1)]} a_{0}, \quad n \in \mathbb{N} .
$$

The above discussion can be summarized in the following result:

Theorem 12 Let $\alpha \in] 0,1\left[, \beta>-, x \in\left[x_{0}, X_{0}\right]\right.$, and $x_{0}, X_{0} \in \mathbb{R}_{+}$such that $\frac{1}{\Gamma(1-2 \alpha)}+\frac{\beta+1}{\Gamma(1-\alpha)}=0$. Then fractional Laguerre equation (10) admits as a particular solution the power series (33), with the coefficients satisfying conditions (35) and given by formula (39).

\section{An operational method}

In this subsection we propose a method to obtain an approximate solution of the fractional Laguerre equation by using the direct and inverse Mellin transforms. Namely, applying Mellin's transform to (10) and taking into account properties (4), we have

$$
\frac{\Gamma(1-s+\alpha)}{\Gamma(1-s-\alpha)} L(s-\alpha)-\frac{\Gamma(1-s)}{\Gamma(1-s-\alpha)} L(s)+\frac{(\beta+1) \Gamma(1-s+\alpha)}{\Gamma(1-s)} L(s-\alpha)+\gamma L(s)=0
$$


Denoting $H(s)=\Gamma(1-s) L(s)$, where $L(s)$ is the Mellin transform of $l(x)$ and

$$
h(x)=\int_{0}^{\infty} e^{-x t} l(t) d t
$$

we get owing to Theorem 1 that $h(x)$ is the inverse Mellin transform of $H(s)$. Thus (40) became

$$
H(s-\alpha)-H(s)+(\beta+1) \frac{\Gamma(1-s-\alpha)}{\Gamma(1-s)} H(s-\alpha)+\gamma \frac{\Gamma(1-s-\alpha)}{\Gamma(1-s)} H(s)=0 .
$$

Taking the inverse Mellin transform we obtain, correspondingly, the equality

$$
x^{-\alpha} h(x)-h(x)+(\beta+1) \int_{0}^{\infty}\left(\frac{x}{t}\right)^{-\alpha} h\left(\frac{x}{t}\right) k(t) \frac{d t}{t}+\gamma \int_{0}^{\infty} h\left(\frac{x}{t}\right) k(t) \frac{d t}{t}=0,
$$

where

$$
k(t)=M^{-1}\left\{\frac{\Gamma(1-s-\alpha)}{\Gamma(1-s)}\right\}=\frac{1}{\Gamma(\alpha)}(t-1)_{+}^{\alpha-1}=\left\{\begin{array}{ll}
\frac{(t-1)^{\alpha-1}}{\Gamma(\alpha)}, & t>1 \\
0, & t<1
\end{array} .\right.
$$

Consider that $h(x)$ admits formal series representation $h(x) \sim \sum_{n=1}^{\infty} b_{n} x^{-\alpha n}$, i.e.,

$$
h(x)=\sum_{n=1}^{N} b_{n} x^{-\alpha n}+O\left(x^{-\alpha N}\right), \quad x \rightarrow \infty, N \in \mathbb{N} .
$$

Substituting (45) into (43) and using Lemma 6 with (44), we come out with the asymptotic equality

$$
\begin{aligned}
x^{-\alpha} \sum_{n=1}^{N} b_{n} x^{-\alpha n}-\sum_{n=1}^{N} b_{n} x^{-\alpha n}+\frac{\beta+1}{\Gamma(\alpha)} & x^{-\alpha} \sum_{n=1}^{N} b_{n} x^{-\alpha n} \int_{1}^{\infty} t^{\alpha n-1}(t-1)^{\alpha-1} d t \\
& -\frac{\gamma}{\Gamma(\alpha)} \sum_{n=1}^{N} b_{n} x^{-\alpha n} \int_{1}^{\infty} t^{\alpha n-1}(t-1)^{\alpha-1} d t=O\left(x^{-\alpha N}\right) .
\end{aligned}
$$

At the meantime, the elementary Beta-integral that appear in (46) is calculated explicitly under condition $\alpha<\frac{1}{N+2}$.

$$
\int_{1}^{\infty} t^{\alpha n-1}(t-1)^{\alpha-1} d t=\frac{\Gamma(\alpha) \Gamma(-\alpha(n+1)+1)}{\Gamma(-\alpha n+1)}, \quad n=0, \ldots, N .
$$

Therefore, substituting (47) into (46), we arrive at the following truncated equation

$$
\begin{aligned}
\sum_{n=2}^{N} b_{n-1} x^{-\alpha n} & -\sum_{n=1}^{N} b_{n} x^{-\alpha n} \\
& +\sum_{n=2}^{N} b_{n-1} \frac{(\beta+1) \Gamma(-\alpha(n+1)+1)}{\Gamma(-\alpha n+1)} x^{-\alpha n}+\sum_{n=1}^{N} b_{n} \frac{\gamma \Gamma(-\alpha(n+1)+1)}{\Gamma(-\alpha n+1)} x^{-\alpha n}=0
\end{aligned}
$$

Collecting the terms which contain equal power of $x$ and equating them to zero, we find

$$
\left\{\begin{array}{l}
b_{1}\left(\frac{\gamma \Gamma(1-2 \alpha)}{\Gamma(1-\alpha)}-1\right)=0 \\
b_{n-1}\left(\frac{(\beta+1) \Gamma(-\alpha(n+1)+1)}{\Gamma(-\alpha n+1)}+1\right)+b_{n}\left(\gamma \frac{\Gamma(-\alpha(n+1)+1)}{\Gamma(-\alpha n+1)}-1\right)=0, \quad n=2,3,4, \ldots, N
\end{array} .\right.
$$

From the first equation of system (48) we conclude that the case $b_{1}=0$ leads to the trivial solution. Hence let consider $\left.b_{1} \neq 0, \gamma \in\right] 0,2[$, and $\left.\alpha \in] 0, \frac{1}{N+2}\right]$ such that

$$
\gamma \Gamma(1-2 \alpha)=1-\alpha
$$


From the second equation of system (48) and taking into account the properties of the Gamma function (see [3]) we have that

$$
b_{n}=-\frac{(\beta+1) \Gamma(-\alpha(n+1)+1)+\Gamma(-\alpha n+1)}{\gamma \Gamma(-\alpha(n+1)+1)-\Gamma(-\alpha n+1)} b_{n-1}, \quad n=2,3,4, \ldots, N,
$$

where numerical simulations indicates that the solutions of equation (49) can not annulate the first term of the right hand side of (50), for any $n=2,3,4, \ldots, N$. Therefore, we can express coefficients $b_{n}$ by the relation

$$
b_{n}=(-1)^{n-1} \prod_{k=2}^{n} \frac{(\beta+1) \Gamma(-\alpha(k+1)+1)+\Gamma(-\alpha k+1)}{\gamma \Gamma(-\alpha(k+1)+1)-\Gamma(-\alpha k+1)} b_{1}, \quad n=2,3,4, \ldots, N .
$$

Since $b_{1} \neq 0$, we get from (48), an approximate solutions of equation (46) in the form $h(x) \sim \sum_{n=1}^{\infty} b_{n} x^{-\alpha n}$. Since $h(x)$ is approximated by the previous series, we use Laplace operational calculus to conclude that equality (41) gives $l(t)$ as a formal series

$$
l(t) \sim \sum_{n=1}^{\infty} \frac{b_{n}}{\Gamma(\alpha n)} t^{\alpha n-1}
$$

The above discussion can be summarized in the following result:

Theorem 13 Let $\left.\left.\left.b_{1} \neq 0, \alpha \in\right] 0, \frac{1}{N+2}\right], \gamma \in\right] 0,2\left[, N \in \mathbb{N}_{0}, x \in\left[x_{0}, X_{0}\right]\right.$, and $x_{0}, X_{0} \in \mathbb{R}_{+}$such that $\gamma \Gamma(1-2 \alpha)=1-\alpha$. Then the fractional Laguerre equation (10) admits as a particular solution the power series (52), with the coefficients given by formula (51).

\section{ACKNOWLEDGMENTS}

This work was supported by Portuguese funds through the CIDMA - Center for Research and Development in Mathematics and Applications, and the Portuguese Foundation for Science and Technology (ŞFCT-Fundação para a Ciência e a Tecnologia $\breve{T}$, within project PEst-OE/MAT/UI4106/2014.

\section{REFERENCES}

1. P.C. Abbot, Generalized Laguerre polynomials and quantum mechanics, J. Phys. A: Math. Gen. 33, 7659 (2000).

2. J. Butzer, Mellin transform theory and the role of its differential and integral operators. Transform Methods and Special Functions, Varna'96 (Conf. Proc.), Bulgarian Acad. Sci., 63-83 (1998).

3. A. Erdelyi, W. Magnus, F. Oberhettinger and F.G. Tricomi, Higher Transcendental Functions-Vol.1,2, Bateman Manuscript Project, McGraw-Hill, New York, 1953.

4. A.A. Kilbas, H. Srivastava and J. Trujillo, Theory and applications of fractional differential equations, North-Holland Mathematics Studies 204, Elsevier, Amsterdam, 2006.

5. M. Klimek and O.P. Agrawal, Fractional Sturm-Liouville problem, Comput. Math. Appl., Part A 66-No.5, 795-812 (2013).

6. F. Mainardi and M. Tomirotti, Seismic pulse propagation with constant Q and stable probability distributions, Annali di Geofisica 40, 1311-1328 (1997).

7. I. Podlubny, Fractional differential equations. An introduction to fractional derivatives, fractional differential equations, to methods of their solutions and some applications, Mathematics in Science and Engineering 198, Academic Press, San Diego, 1999.

8. A.P. Prudnikov, Yu.A. Brychkov and O.I. Marichev, Integrals and Series, Volume 3: More special functions, Gordon and Breach Publisher, New York, 1990.

9. M.M. Rodrigues and N. Vieira, On fractional Whittaker equation and operational calculus, J. Math. Sci. Univ. Tokyo 20, 1-20 (2013).

10. M.M. Rodrigues, N. Vieira and S. Yakubovich, "Operational Calculus for Bessel's Fractional Equation," in Advances in Harmonic Analysis and Operator Theory, Operator Theory: Advances and Applications - Vol.229, edited by J. Almeida, L. Castro and F. Speck; Birkhäuser, Basel, 2013, 351-364.

11. S. G. Samko, A.A. Kilbas and O. I. Marichev, Fractional integrals and derivatives: theory and applications, Gordon and Breach, New York, 1993.

12. E.C. Titchmarsh, Introduction to the theory of Fourier integrals, Claredon Press, Oxford, 1948. 